\title{
Plataformas de livros acadêmicos em acesso aberto e sua representação no Twitter: métricas de divulgação, descoberta e avaliação
}

\author{
Platforms for scholarly books in open access \\ and their representation on Twitter: metrics \\ of disclosure, discovery, and evaluation
}

Nanci Elizabeth ODDONE ID 0000-0002-0086-5253
Cláudio Márcio de FRANÇA' iD 0000-0002-6440-4421

\section{Resumo}

O presente artigo explora o desempenho no Twitter de quatro plataformas que publicam, agregam e disseminam livros acadêmicos em acesso aberto: Scientific Electronic Library Online-Livros, Directory of Open Access Books, Open Access Publishing in European Networks e OpenEdition Books. Os autores acompanharam as postagens dessas instituições na rede social Twitter entre os dias 8 e 18 de julho de 2019, mapeando os indicadores gerados por cada uma delas. O objetivo foi avaliar se as métricas de atenção e influência observadas podem ampliar a circulação e o alcance dos livros acadêmicos em acesso aberto, contribuindo para a sua divulgação, descoberta e avaliação. Para interpretação das métricas geradas no Twitter fez-se uso das ferramentas estatísticas Follor.me e TweetReach, cuja aplicação fornece um relatório analítico que revela o engajamento, as impressões e o alcance real e potencial de cada perfil. Após a verificação dessas métricas, investigou-se também as experiências dos usuários e as implicações geradas pelo uso, objetivando compreender as possíveis reações dos seguidores desses perfis sobre o conteúdo divulgado pelas plataformas. Os resultados mostraram que as postagens realizadas pelas plataformas visaram prioritariamente à promoção e divulgação. As manifestações dos seguidores, por sua vez, reverberando as postagens por meio de réplicas e respostas, potencializam os efeitos de uso, repercussão e influência, conjuntura que embora não ateste a qualidade do conteúdo, fornece importantes indícios sobre o público leitor, auxiliando ainda os editores na identificação de temas emergentes e na avaliação das estratégias de divulgação dos livros acadêmicos em acesso aberto.

Palavras-chave: Acesso à informação. Bibliometria. Livros. Redes sociais (em linha).

\begin{abstract}
This article explores the Twitter performances of four platforms that publish, aggregate, and disseminate open access academic books: Scientific Electronic Library Online-Livros, Directory of Open Access Books, Open Access Publishing in European Networks, and

1 Universidade Federal do Estado do Rio de Janeiro, Centro de Ciências Humanas, Programa de Pós-Graduação em Biblioteconomia. Av. Pasteur, 458, Praia Vermelha, 22290-255, Rio de Janeiro, RJ, Brasil. Correspondência para/Correpondence to: N.E. ODDONE.E-mail:<neoddone@unirio.br>.

Este artigo é uma versão ampliada e consideravelmente revista da comunicação oral apresentada pelos autores no "I Congresso de Altmetria e Ciência Aberta da América Latina (LATmetrics)". Universidade Federal Fluminense, 2018.

Recebido em 18 de fevereiro de 2019, versão final reapresentada em 17 de junho de 2019 e aprovado em 29 de agosto de 2019.

Como citar este artigo/How to cite this article

Oddone, N.E; França, C.M. Plataformas de livros acadêmicos em acesso aberto e sua representação no Twitter: métricas de divulgação, descoberta e avaliação. Transinformação, v.31, e190011, 2019. http://dx.doi.org/10.1590/2318-0889201931e190011
\end{abstract}


OpenEdition Books. We followed these institutions' posts on Twitter between July 8 and 18, 2019, mapping their indicators. The objective was to evaluate whether the observed metrics of attention and influence could increase the circulation and reach of academic books in open access, contributing to their dissemination, discovery, and evaluation. To interpret the metrics generated on Twitter, we used the statistical tools Follor.me and TweetReach, which provide analytical reports that reveal each profile's engagement, perceptions and its actual and potential reach. After verifying these metrics, we also investigated users' experiences and the implications of the use, aiming to understand the range of possible reactions followers had regarding the content the platforms disclosed. The results showed that the posts aimed primarily at promotion and dissemination. Followers' manifestations, in turn, reverberate the posts through replicas and responses, maximizing the effects of use, repercussion, and influence. While that does not attest to the quality of the content, it provides important indications about the readership, helping editors identify emerging themes and evaluate strategies for the dissemination of academic books in open access.

Keywords: Access to information. Bibliometrics. Books. Social media (online).

\section{Introdução}

Ao longo das últimas décadas as atividades de comunicação científica vêm sendo irreversivelmente alteradas pela introdução de uma variedade de tecnologias eletrônicas na cadeia de produção, circulação e uso do conhecimento (Bosman; Kramer, 2015). De um passado nem tão remoto - representado pelo consagrado modelo de comunicação científica delineado nos estudos de Garvey e Griffith (1971), cujo processo linear constitui-se de etapas segmentadas e consecutivas a serem percorridas -, até o presente, onde novos atores humanos e não humanos integram as redes de produção e disseminação dos resultados da ciência (Oddone et al., 2000), percorreu-se um caminho marcado por significativas transformações operacionais e comportamentais. Um dos efeitos mais visíveis dessas mudanças é a velocidade com que são disseminadas as descobertas e os avanços científicos, que fluem sem cessar pelas redes do ciberespaço, concebido como o"meio de comunicação que surge da interconexão mundial de computadores" (Levy, 1999, p.17).

Embora as primeiras alterações no modelo tradicional de comunicação científica tenham surgido ainda na década de 70 do século XX, envolvendo não só o uso de formatos digitais, mas também outras iniciativas, como o auto-arquivamento de pré-prints adotado por algumas áreas (Symplectic, 2014), foi a partir dos primeiros anos do século XXI que se observou o progressivo engajamento de editores e veículos acadêmicos ao movimento de acesso aberto proposto na Declaração de Budapeste (Suber, 2015). Entre as consequências da sistemática difusão das ideias do acesso livre à informação científica registra-se a pressão exercida sobre as grandes editoras internacionais no sentido da modernização da cadeia produtiva da publicação acadêmica, destacando-se a migração do formato impresso para o formato digital, o que estimulou "mudanças tanto no mercado editorial da comunicação científica como nas estratégias de visibilidade do conhecimento" (Gruszynski; Golin, 2006, p.2).

A conversão dos periódicos científicos impressos para o formato eletrônico e o sucessivo desenvolvimento das bases de dados como canais preferenciais para reunir e disponibilizar todo o conteúdo bibliográfico e referencial, corrente e retrospectivo, agilizou e ampliou o acesso à produção científica qualificada. Recentemente, a chegada de novos atores, como os agregadores de conteúdo, os repositórios e as mídias sociais, tem promovido o alargamento desse cenário, potencializando o acesso e o uso do periódico científico. Se por um lado esse fenômeno favoreceu a inclusão de outras fontes e suportes informacionais além do periódico científico, por outro lado concorreu para uma crescente vulnerabilidade e dispersão dos produtos editorais, dificultando a localização e recuperação da informação científica relevante.

Os livros acadêmicos não passaram incólumes pelas transformações nascidas do ambiente digital. No entanto, como não integram o mainstream das atividades de comunicação científica ligadas às Ciências Naturais e Exatas - que têm o periódico como o principal veículo de disseminação dos resultados científicos -, os estudos sobre o livro e as reflexões sobre as novas práticas de disseminação, circulação e avaliação em ambiente digital têm se mostrado ainda bastante embrionárias, com raros registros na literatura. Apesar disso, é comum encontrar 
referências à relevância do livro como canal de circulação do conhecimento científico, sobretudo quando se trata das Ciências Sociais e Humanas, cujos hábitos de leitura e citação - que se valem mais de livros e capítulos de livros do que revistas científicas -, são reconhecidamente distintos daqueles apresentados nas áreas mais "duras" da ciência (Hicks, 2004; Mueller, 2005; Meadows, 2009).

O desenvolvimento de bibliotecas digitais, repositórios, editoras e plataformas especializadas cujo objetivo prioritário é reunir livros acadêmicos em variados formatos digitais de acesso aberto, tem contribuído para atenuar o cenário de dispersão já assinalado e, ao mesmo tempo, para "maximizar a visibilidade, acessibilidade, uso e impacto das pesquisas, ensaios e estudos" (Scientific Electronic Library Online Livros, 2012, online) publicados como obras monográficas ou coletâneas. No contexto desse movimento de inovação editorial (Dourado, 2012) que fortalece o acesso livre ao conhecimento no segmento do livro acadêmico encontram-se quatro iniciativas significativas, que incluem: Scientific Electronic Library Online Livros (SciELO-Livros), Open Access Publishing in European Networks (OAPEN), Directory Open Access Books (DOAB) e OpenEdition Books.

Ao mesmo tempo que promovem o acesso a uma vasta produção livresca em diferentes áreas do conhecimento, essas iniciativas permitem também avaliar como a comunidade científica está recepcionando esse conteúdo. Tradicionalmente, uma das principais formas de avaliar o impacto alcançado por uma publicação científica é por meio das citações que a mesma recebe. No entanto, citações não podem ser tomadas como únicas fontes qualificadoras, pois além do impacto propriamente acadêmico a atividade científica contribui também para o desenvolvimento social, educacional e cultural, o que deve ser igualmente considerado em processos de avaliação.

Em meio ao desafio de encontrar qualificadores melhores e mais precisos para os produtos científicos, a web social tem contribuído significativamente para ampliar o acesso aos resultados das pesquisas, que têm circulado muito além dos muros da academia. A partir da contínua interação entre autores e leitores através das mídias sociais, redes acadêmicas e ferramentas usadas para criar, coletar, compartilhar, organizar e gerenciar informações, tem sido possível produzir indicadores e métricas alternativas (altmetrics), cujo objetivo é monitorar o impacto e a influência da ciência para além da comunidade de pares e do ambiente científico dos laboratórios e centros de pesquisa (Bonnet; Méndez-Brandy, 2017).

Considerando o livro como um veículo que dissemina conteúdos diversificados, registrando não só as descobertas que se tornaram fatos científicos (Latour, 1999) mas também conteúdos relacionados à arte, à cultura, ao ensino e ao lazer, admite-se que a adoção de métricas alternativas para aferir sua qualidade e sua repercussão representa um complemento vantajoso para os tradicionais indicadores baseados em citações. Variáveis tradicionais atribuídas aos livros, como volume de vendas e autoridade do autor também poderiam ser avaliadas pelas novas métricas, que têm potencial para ampliar a circulação de monografias e coletâneas (Torres-Salinas; Robinson-Garcia; Gorraiz, 2017).

Alguns dos indicadores obtidos junto às mídias sociais emergiram inicialmente como recursos de marketing digital no ambiente corporativo. No entanto, em face do crescimento da ciência e da necessidade de divulgar e disseminar a extensa produção acadêmica para além dos tradicionais canais formais de comunicação científica, vislumbra-se nas mídias sociais um ambiente privilegiado para o desenvolvimento de atividades de divulgação e avaliação, obtendo dados auxiliares e complementares, que permitam a visualização mais precisa do impacto e da popularidade de uma publicação.

Situada neste contexto, a presente pesquisa propõe identificar que indicadores de descoberta, divulgação e avaliação dos livros publicados em acesso aberto por plataformas cooperativas podem ser obtidos a partir das métricas de atenção, engajamento e influência geradas na rede social Twitter. Acredita-se que o momento é oportuno para a realização de um estudo dessa natureza, tendo em vista a existência de esforços convergentes para o desenvolvimento de editoras, plataformas e bases de dados que privilegiam o livro digital em acesso aberto. 
Indicadores de reconhecimento e de impacto social dessas obras podem ser empregados não só para a avaliação da atividade e da produção científicas, mas também para a formulação das estratégias de divulgação das editoras universitárias e comerciais.

\section{Procedimentos Metodológicos}

De caráter exploratório, a presente pesquisa adotou duas perspectivas distintas para coletar e analisar os dados. Inicialmente empregou-se uma estratégia quantitativa e estatística para contabilizar os indicadores e as métricas de atenção Online e, mais adiante, uma estratégia qualitativa, utilizando análise de conteúdo, para classificar o corpus de postagens de acordo com as categorias analíticas criadas por Araujo e Furnival (2016). Privilegiou-se o Twitter como universo empírico da pesquisa, de modo que se pudesse acompanhar e explorar as postagens, observando os indicadores e as métricas produzidas pelas quatro plataformas de livros acadêmicos em acesso aberto selecionadas: SciELO Livros, DOAB, OAPEN e OpenEdition Books. Ao contrário das outras três plataformas, a SciELO Livros não se limita a disponibilizar obras em acesso aberto, pois aproximadamente um terço do seu acervo tem caráter comercial, oferecendo apenas a opção de aquisição do conteúdo.

A opção pelo Twitter não ocorreu de forma aleatória, mas fundamentou-se em evidências registradas na literatura, como os estudos de Fausto, Leite-Silva e Ferreira (2015), que consideram essa mídia social como um importante canal para as práticas de comunicação científica. Além de Gouveia (2013), Priem, Piwowar e Hemminger (2012) igualmente destacam o potencial do Twitter para facilitar contatos informais em torno de artigos, pesquisas e conferências, para monitorar indicações e estimular interações entre pesquisadores, configurando-se como uma ferramenta de uso recorrente que também oportuniza a obtenção de documentos de interesse.

O estudo concentrou-se inicialmente na interpretação das métricas geradas no Twitter. As postagens realizadas pelos quatro perfis selecionados foram contabilizadas e examinadas por meio de duas ferramentas estatísticas independentes: o Foller.me (https://foller.me/) e o TweetReach (https://tweetreach.com/), que disponibilizam essas informações de forma gratuita na Web, sendo necessário apenas a indicação do nome do usuário, antecedido pelo sinal gráfico arroba. Os dados foram coletados no período de dez dias entre 8 e 18 de julho de 2018. A abrangência do estudo limitou-se a esses dez dias em razão das ferramentas estatísticas utilizadas não permitirem a análise por um período maior de tempo.

Embora o ambiente das mídias sociais propicie múltiplas reflexões a partir de dados como postagens, compartilhamentos, respostas, menções e exame de termos, frases, Uniform Resource Locator (URL), handles e hashtags citados, o relatório analítico gerado pelas ferramentas adotadas, com dados que já se encontram previamente definidos, é específico de cada perfil e envolve variáveis como total de seguidores, número de postagens e atividades, bem como as métricas de alcance, engajamento e impressão, que são as unidades de análise mais comumente utilizadas em estudos sobre métricas em redes sociais.

O indicador de alcance é associado à visibilidade, materializada pelo número de seguidores que um perfil possui em uma rede social (Araújo, 2018). Nesta pesquisa, para compreender o alcance de cada perfil, tomou-se a quantidade de pessoas que efetiva ou potencialmente leram ou visualizaram uma mensagem (Silva, 2013). As atividades de interação observadas nas redes sociais são determinantes para aferição da métrica de engajamento, que permite vislumbrar o total de vezes que seguidores interagiram de algum modo com o perfil e com o conteúdo publicado (Twitter, 2018). Nesse estudo, foram consideradas como interações as atividades de curtidas, respostas a postagem e as réplicas, com ou sem comentários. A impressão é uma métrica de exposição contígua ao alcance, diferindo dessa por ser mais abrangente, já que em seu espectro de análise inclui não só os usuários únicos diretamente atingidos por uma mensagem, mas também os que potencialmente podem ter sido alcançados. Sua representação se dá pela soma simples de todas as audiências, independente das formas de acesso e, principalmente, da frequência de visualização (Silva, 2013). 
A segunda etapa do estudo concentrou-se em investigar as experiências que os usuários podem obter e as implicações geradas pelo uso. Para tanto recorreu-se à metodologia adotada por Araújo e Furnival (2016), que sistematizaram seis categorias para analisar conteúdos científicos mencionados em redes sociais, como se vê no Quadro 1. Embora o universo empírico de Araújo e Furnival tenha envolvido apenas artigos científicos, considerouse adequado adotar as mesmas categorias no presente estudo, de forma a compreender a interação e as possíveis reações dos seguidores ao conteúdo divulgado por essas plataformas de livros digitais em acesso aberto, além de capturar possíveis métricas de atenção e influência dos produtos por elas disponibilizados.

Para proceder a essa classificação adotou-se a técnica da análise de conteúdo sobre toda a atividade produzida na timeline dos quatro perfis durante o período estudado, integrando nesse corpus as postagens (tweets), as respostas (replies), as menções e as réplicas (retweets). Constatou-se que o cenário observado no Twitter, por suas características, permite vislumbrar outras métricas possíveis, surgidas a partir da relação das editoras com os usuários. A interpretação dos dados envolvidos nesse segundo momento da pesquisa ocorreu sem o emprego de qualquer meio externo, sendo as reflexões e associações produzidas por meio da leitura, contextualização e categorização dos tweets.

\section{Resultados e Discussão}

O Complete Guide to Twitter Analytics (Simply Mensured, 2014) considera o Twitter como um campo fértil para a prospecção de análises métricas e estatísticas. Dois elementos estruturais são necessários para explorar todo o potencial dessa rede social na geração de informações sobre determinado perfil: o número de seguidores a ele vinculados e a frequência das postagens e da inserção de novos tweets. Nesse contexto, as primeiras variáveis verificadas no presente estudo foram os seguidores e as postagens de cada plataforma (Tabela 1).

Ainda que os quatro perfis atinjam um público considerável, a disparidade entre os que possuem o maior e o menor número de seguidores, mesmo que não seja determinante, pode ter sofrido influência da data de criação do perfil na rede social. Para comparação, registre-se que a conta da OpenEdition Books no Twitter foi criada em 2009, enquanto que a do DOAB teve início no ano de 2012.

A Tabela 1 indica também a quantidade total de postagens realizadas desde a criação do perfil no Twitter até o período de coleta dos dados. Considerando que todos os perfis estudados estão em atividade há quase dez anos, deduz-se que as práticas de divulgação das quatro plataformas são incipientes e irregulares já que a média de postagens alcança apenas um ou menos de um tweet por dia.

Quanto à correspondência entre seguidores e postagens, observa-se que o SciELO Livros apresentou, proporcionalmente, indicadores menos expressivos no que se refere ao potencial comunicativo da rede social, pois embora possua o segundo maior contingente de seguidores, é o perfil que menos disponibiliza conteúdo em termos absolutos e relativos.

Quadro 1. Categorias para análise de contexto de postagens em redes sociais.

\begin{tabular}{ll}
\hline Categoria de Análise & Descrição \\
\hline Compartilhamento (repasse) & Copia o tweet, sintetizando a mensagem publicada. \\
Explicativo & Detalha e inclui dados complementares à mensagem publicada. \\
Conclusivo & Estabelece credibilidade e qualifica a mensagem publicada. \\
Argumentativo & Argumenta e/ou discute a mensagem contida na publicação. \\
Exortativo & Vale-se da mensagem publicada para sensibilizar práticas e ações. \\
Problematizante & Utiliza críticas ou desqualifica a mensagem publicada. \\
\hline
\end{tabular}

Fonte: Adaptado de Araújo e Furnival (2016). 
Tabela 1. Número total de seguidores e postagens até 18 de julho de 2018.

\begin{tabular}{lccc}
\hline Plataforma - Criação do Perfil & Seguidores & Postagens & Média de postagens por ano/por dia \\
\hline OpenEdition Books - 2009 & 9.632 & 3.539 & $393,22 / 1,07$ \\
SciELO Livros - 2011 & 9.29 & 619 & $88,42 / 0,24$ \\
OAPEN Books - 2010 & 4.296 & 2.551 & $318,87 / 0,87$ \\
DOAB - 2012 & 2.8 & 1.65 & $275,00 / 0,75$ \\
\hline
\end{tabular}

Nota: DOAB: Directory Open Access Books; OAPEN: Open Access Publishing in European Networks; SciELO Livros: Scientific Electronic Library Online Livros. Fonte: Elaborada pelos autores (2018) com base em dados coletados.

Examinando as postagens (tweets) e as atividades relacionadas, como réplicas (retweets) e respostas (replies), a Tabela 2 sintetiza as ações empreendidas pelas plataformas durante o período do presente estudo.

Em consonância com o maior número de postagens realizadas entre os dias 8 e 18 de julho de 2018, caracterizando uma deliberada estratégia de divulgação de seus produtos, a plataforma OpenEdition Books apresentou o maior número global de atividades derivadas, com destaque para a quantidade de réplicas, que representam o endosso e a recomendação dos seguidores aos conteúdos que atendem às suas necessidades informacionais (Recuero; Zago, 2010). No que se refere ao reduzido número de respostas e comentários obtidos pelas postagens, sobretudo quando se relaciona esse dado à quantidade de seguidores de cada perfil, acredita-se que seria imprudente traçar conclusões, tendo em vista que o curto período de coleta é uma das limitações do presente estudo.

Como anteriormente destacado, as métricas mais usuais no âmbito do Twitter são as que medem o alcance, o engajamento e as impressões. Os dados coletados sobre essas variáveis são apresentados na Tabela 3.

A primeira interpretação obtida pela análise dos dados da Tabela 3 enfatiza a relação entre o número de seguidores e a quantidade de contas alcançadas pelas mensagens publicadas. Quando considerados os perfis da OpenEdition Books, da OAPEN Books e do DOAB constata-se que seu alcance foi aproximadamente dez vezes

Tabela 2. Número de postagens e atividades derivadas entre 8 e 18 de julho de 2018.

\begin{tabular}{lcccc}
\hline Atividades no Twitter & OpenEdition Books & OAPEN Books & DOAB & SciELO Livros \\
\hline Postagens & 26 & 13 & 6 & 7 \\
Réplicas & 66 & 34 & 30 & 19 \\
Respostas & 8 & 10 & 12 & 0 \\
\hline
\end{tabular}

Nota: DOAB: Directory Open Access Books; OAPEN: Open Access Publishing in European Networks; SciELO Livros: Scientific Electronic Library Online Livros.

Fonte: Elaborada pelos autores (2018) com base nos dados coletados.

Tabela 3. Métricas alcançadas pelas plataformas de livros eletrônicos em acesso aberto.

\begin{tabular}{lccc}
\hline \multirow{2}{*}{ Plataformas } & \multicolumn{1}{c}{ Métricas } \\
\cline { 2 - 4 } & Alcance Potencial & Engajamento & Impressões potenciais \\
\hline OpenEdition Books & 80.892 & 87 & 96.501 \\
SciELO Livros & 47.694 & 17 & 120.09 \\
OAPEN Books & 43.32 & 36 & 105.255 \\
DOAB & 28.877 & 30 & 63.912 \\
\hline
\end{tabular}

Fonte: Elaborada pelos autores (2018) com base nos dados coletados. 
superior ao total de seus seguidores. O alcance da plataforma SciELO Livros, ao contrário, limita-se à metade dessa taxa, embora em números absolutos a plataforma seja a segunda com maior alcance potencial. Considerando que as ações com maior alcance estão relacionadas às postagens de divulgação e direcionamento de conteúdo (Macedo, 2014), verifica-se que os três primeiros perfis comunicam-se de forma mais rotineira com seus seguidores e com um público potencial mais amplo.

Os dados sobre engajamento produzidos pelos quatro perfis selecionados, por sua vez, demonstram que, quando comparada às demais plataformas, a OpenEdition Books obteve um número superior de interações com seus seguidores e com outros usuários do Twitter durante o período estudado. Essa métrica resulta não só do maior número de seguidores como da maior quantidade de postagens dessa plataforma, constituindo indício seguro do envolvimento e da participação do público em geral com o conteúdo disseminado pelas mensagens divulgadas (Silva, 2013). O perfil do SciELO Livros, em contrapartida, caracteriza baixo engajamento, decorrente da menor quantidade de postagens e do número reduzido de interações, que impossibilitam uma maior interação com os usuários. Tal fato tende a repercutir negativamente na métrica de engajamento, pois quando a produção e o direcionamento de conteúdo oscilam, a percepção dos produtos e da marca e a penetração no mercado acabam por afastar os seguidores do perfil (Simply Measured, 2014).

A taxa de impressões potenciais representa o grau de exposição alcançado por uma marca e seus produtos, ou seja, o número de vezes que os mesmos são incluídos em postagens. Nesse sentido, segundo as evidências obtidas neste estudo, o cálculo das impressões avalia melhor as estratégias de descoberta do que as de divulgação, pois o aumento no número de impressões é influenciado diretamente pela estrutura da postagem. Elementos como o título, a estrutura simples e sintética da mensagem comunicada e a inclusão de identificadores persistentes, que possibilitem o acesso imediato ao conteúdo - como o Digtial Object Identifier (DOI) e o handle -, são atributos que levam ao consumo da informação veiculada, potencializando o processo de descoberta. A despeito de ter apresentado o menor índice de engajamento e o menor número de postagens, o perfil do SciELO Livros no Twitter exibe a maior quantidade de impressões, atestando que não existe simetria entre as métricas de engajamento e de exposição e que não se deve confundir as impressões com a quantidade de vezes que os tweets são vistos ou lidos (Twitter, 2018).

O segundo momento da pesquisa envolveu a classificação, de acordo com as categorias analíticas de Araújo e Furnival (2016), das réplicas (retweets) e das respostas (replies) postadas pelos seguidores dos quatro perfis em interação com as postagens realizadas por cada plataforma. O exame desse corpus possibilitou observar as reações dos seguidores (Tabela 4).

A análise dos dados permitiu observar que predominou o compartilhamento das postagens realizadas pelas plataformas de livros digitais no Twitter, o que, respeitadas as diferenças entre as duas pesquisas, vai ao encontro

Tabela 4. Classificação das réplicas e respostas segundo categorias analíticas.

\begin{tabular}{|c|c|c|c|c|c|c|c|c|}
\hline \multirow{2}{*}{ Categoria Analítica } & \multicolumn{2}{|c|}{ OpenEdition Books } & \multicolumn{2}{|c|}{ OAPEN BOoks } & \multicolumn{2}{|c|}{$\mathrm{DOAB}$} & \multicolumn{2}{|c|}{ SciELO Livros } \\
\hline & $\mathrm{n}$ & $\%$ & $\mathrm{n}$ & $\%$ & $\mathrm{n}$ & $\%$ & $n$ & $\%$ \\
\hline Compartilhamento (repasse) & 56 & 75,50 & 40 & 90,90 & 40 & 95,25 & 19 & 100,00 \\
\hline Explicativo & 5 & 6,75 & 2 & 4,55 & 2 & 4,75 & & \\
\hline Conclusivo & 3 & 4,25 & \multicolumn{2}{|c|}{0} & \multicolumn{2}{|c|}{0} & \multicolumn{2}{|c|}{0} \\
\hline Argumentativo & 10 & 13,50 & 2 & 4,55 & & & & \\
\hline Exortativo & \multicolumn{2}{|c|}{0} & \multicolumn{2}{|c|}{0} & \multicolumn{2}{|c|}{0} & \multicolumn{2}{|c|}{0} \\
\hline Problematizante & \multicolumn{2}{|c|}{0} & \multicolumn{2}{|c|}{0} & \multicolumn{2}{|c|}{0} & \multicolumn{2}{|c|}{0} \\
\hline
\end{tabular}

Fonte: Elaborado pelos autores (2018) a partir de Araújo e Furnival (2016), com base nos dados coletados 
das conclusões do estudo de Araújo e Furnival (2016), caracterizando essa mídia social como um espaço proveitoso para práticas de divulgação editorial, com vistas à promoção das plataformas, como se constata na postagem da @SciELOLivros: "chegamos aos 1.000 títulos publicados" (SciELO Books, 2018), que deu origem a todas as réplicas e repostas examinadas por esta pesquisa.

Além da promoção de uso, o incentivo para que novos autores e editores se associem à iniciativa de edição em acesso aberto também foi verificado, como se pode constatar na postagem de @TextandAcademic: "DOAB está aberto a todos os editores que publicam livros acadêmicos revisados por pares em acesso aberto [...]"2 (Text \& Academic Authors, 10 jul., 2018, tradução nossa). Por outro lado, o reduzido número de mensagens que podem ser encaixadas nas outras categorias de análise expressa a complexidade de construir contextos avaliativos tomando por base as respostas e as réplicas, tal como ficou evidenciado no estudo de Hammarfelt (2014), que adverte sobre a inconsistência de considerar a audiência como um reconhecimento tácito de qualidade.

\section{Conclusão}

O panorama de crescente influência das métricas alternativas e sociais autoriza novas formas de mensurar o impacto da produção científica em meio digital a partir das interações geradas nas redes sociais. Em relação ao livro científico, cujas tradicionais práticas de avaliação permaneceram, até recentemente, mais qualitativas que quantitativas, mais tácitas e consensuais que objetivas, essas ferramentas suplementares de divulgação e avaliação de obras publicadas em acesso aberto mostram-se promissoras. O presente estudo confirmou a aplicabilidade das métricas alternativas aos livros acadêmicos publicados em acesso aberto, ratificando a importância desses novos indicadores de qualidade no contexto científico. Identificou-se também o interesse das plataformas que gerenciam essas publicações acadêmicas em ampliar o circuito de divulgação desses novos recursos informacionais.

Os resultados da pesquisa mostraram que número considerável das postagens realizadas pelas plataformas destinam-se à promoção e à divulgação, confirmando as conclusões de muitos autores. Além disso, as manifestações por parte dos seguidores, majoritariamente reverberando as postagens por meio de réplicas e respostas, potencializam os efeitos de uso, repercussão e influência, conjuntura que embora não ateste a qualidade do conteúdo, fornece importantes indícios sobre o público leitor, auxiliando ainda os editores na identificação de temas emergentes e na avaliação das estratégias de divulgação dos livros acadêmicos em acesso aberto.

No que tange ao objetivo proposto, pode-se afirmar que esta pesquisa foi bem-sucedida. Deve-se reconhecer, porém que a reduzida amostra de dados obtida através dos aplicativos Foller.me e TweetReach representa uma limitação, já que não permite generalizações. Espera-se, em estudos futuros, realizar análise semelhante sobre um volume maior de dados, de forma a explorar novos cenários, em especial no contexto das respostas às postagens que, denotando algum tipo de juízo, podem fornecer novas perspectivas para avaliar o impacto de determinadas obras ou temáticas.

Por fim, visando obter uma perspectiva comparada dos resultados alcançados neste estudo, sugere-se que novas pesquisas sejam desenvolvidas em outras redes sociais, em particular aquelas voltadas para pesquisadores, como ResearchGate, Academia.edu e Mendeley, a fim de identificar quais as métricas e indicadores gerados e como estes podem apoiar as estratégias de descoberta, divulgação e avaliação de livros acadêmicos.

\section{Colaboradores}

Todos os autores contribuíram na concepção e no desenho do estudo, na análise de dados e na redação final.

${ }^{2}$ DOAB open to all publishers who publish academic, peer reviewed books in Open Access (Text \& Academic Authors, 2018) 


\section{Referências}

Araújo, R.F. Marketing científico digital e métricas de mídias sociais: indicadores-chave de desempenho de periódicos no Facebook. Informação e Sociedade: Estudos, 28, n.1, p.7-22, 2018. Disponível em: http://www.periodicos.ufpb.br/index. php/ies/article/view/22063. Acesso em: 2 jun. 2019.

Araújo, R.F.; Furnival, A.C.M. Comunicação científica e atenção online: em busca de colégios virtuais que sustentam métricas alternativas. Informação e Informação, v. 21, n.2, p.68-89, 2016. Disponível em: http://www.uel.br/revistas/uel/index.php/ informacao/article/view/27297. Acesso em: 28 dez. 2018.

Bonnet, J.L.; Méndez-Brady, M. Making the mission visible: altmetrics and nontraditional publishing. Digital Library Perspectives, v.33, n.4, p.294-304, 2017. Available from: https:// www.emeraldinsight.com/doi/abs/10.1108/DLP-01-2017-00 02. Cited: Jan. 2, 2019.

Bosman, J.; Kramer, B. 101 innovations in scholarly communication: how researchers are getting to grip with the myriad of new tools. LSE Impact of Social Sciences Blog, 11 Nov. 2015. Available from: https://blogs.Ise.ac.uk/impact ofsocialsciences/2015/11/11/101-innovations-in-scholarlycommunication/. Cited: June 7, 2019.

Dourado, S.M. Identificando a inovação editorial na cadeia produtiva do livro universitário brasileiro. 2012. 110f. Dissertação (Mestrado em Ciência da Informação) - Universidade Federal da Bahia, Salvador, 2012.

Fausto, S.; Leite-Silva, S.; Ferreira, S.M.S.P. Twitter como backchannel de eventos científicos compartilhados na web social: um novo canal informal de comunicação científica. Ciência da Informação em Revista, v.2, n.1, p.55-61, 2015. Disponível em: http://www.seer.ufal.br/index.php/cir/article/ view/1707. Acesso em: 3 jan. 2019.

Garvey, W.D.; Griffith, B.C. Scientific communication: its role in the conduct of research and creation of knowledge. American Psychologist, v.26, n.4, p.349, 1971. Available from: https:// psycnet.apa.org/doiLanding?doi=10.1037\%2Fh0032059. Cited: Jan. 5, 2019.

Gouveia, F.C. Altmetria: métricas de produção científica para além das citações. Liinc em Revista, v.9, n.1, p.214-227, 2013. Disponível em: http://revista.ibict.br/liinc/article/view/3434. Acesso em: 3 jan. 2019.

Gruszynski, A.C; Golin, C. Periódicos científicos nos suportes impresso e eletrônico: apontamentos para um estudo-piloto na UFRGS. Revista EPTIC, v.8, n.2, p.1-15, 2006. Disponível em: https://seer.ufs.br/index.php/eptic/article/view/285. Acesso em: 28 dez. 2018.

Hammarfelt, B. Using altmetrics for assessing research impact in the humanities. Scientometrics, v.101, n.2, p.1419-1430, 2014. Available from: https://link.springer.com/article/10.1007/s111 92-014-1261-3. Cited: June 2, 2019.

Hicks, D. The four literatures of Social Science. In: MOED, H. (Org.). Handbook of quantitative science and technology research. Dordrecht: Springer, 2004. p.473-496. Available from: http://yunus.hacettepe.edu.tr/ tonta/courses/spring2011/ bby704/Handbook\%20of\%20Quantitative\%20Science\%20 and\%20Technology\%20Research.pdf\#page=467. Cited: Jan. 5, 2019.
Latour, B. Ciência em ação: como seguir cientistas e engenheiros sociedade afora. São Paulo: Unesp, 1999.

Levy, P. Cibercultura. São Paulo: Editora 34, 1999. p.17.

Macedo, T. Métricas de marketing digital e sua aplicação nas ações de marketing das organizações: estudo de caso múltiplos. Porto Alegre, 2014. 119f. Dissertação (Mestrado em Administração) - Universidade Federal do Rio Grande do Sul, Porto Alegre, 2014. Disponível em: https://lume.ufrgs.br/ handle/10183/101499. Acesso em: 2 jun. 2019.

Meadows, A.J. A comunicação científica. Brasília: Briquet de Lemos, 2009.

Mueller, S.P.M. A publicação da ciência: áreas científicas e seus canais preferenciais. Datagramazero, v.6, n.1, p.1-13, 2005. Disponível em: http://repositorio.unb.br/bitstream/10 482/980/2/ARTIGO_PublicacaoCiencia.pdf. Acesso em: 28 dez. 2018.

Oddone, N.E. et al. Centros de cálculo: a mobilização do mundo. Informare: Cadernos do ProgramadePós-Graduação em Ciência da Informação, v.6, n.1, p.29-43, 2000. Disponível em: http://www.brapci.inf.br/index.php/article/download/15151. Acesso em: 20 jan. 2019.

Priem, J.; Piwohar, H.A.; Hemminger, B.M. Altmetrics in the wild: using social media to explore scholarly impact. [S.I]: ArXiv, 2012. Preprint, p.1-17, mar. Available from: https://arxiv. org/abs/1203.4745. Cited: Jan. 3, 2019.

Recuero, R.; Zago, G. "RT, por favor": considerações sobre a difusão de informações no Twitter. Fronteiras: Estudos Midiáticos, v.12, n.2, p.69-81, 2010. Disponível em: http://www. revistas.unisinos.br/index.php/fronteiras/article/view/4668. Acesso em: 2 jun. 2019.

SciElO Books. Chegamos aos 1.000 títulos publicados. [São Paulo], 18 jul. 2018. Twitter: @SciELOBooks. Disponível em: https://twitter.com/SciELOBooks/status/101955648344341 2993?s=20. Acesso em: 27 maio 2019.

Scientific Eletronic Library Online Livros. Introdução. São Paulo: SciELO, 2012. Disponível em: http://books.scielo.org/ introducao/. Acesso em: 15 jan. 2019.

Silva, T. Métricas e monitoramento de mídias sociais. [S.I.]: SlideShare, 2013. 228 slides. Disponível em: http:// pt.slideshare.net/tarushijio/monitoramento-de-mdiassociais-imerso-digital. Acesso em: 2 jun. 2019.

Simply Measured. The complete guide to Twitter analytics: how to analyze the metrics that matter. [S.I.]: Simple Measured, 2014. Available from: https://www-users.cs.york.ac.uk/ alistair/research/Social\%20media/CompleteGuidetoTwitter AnalyticsSimplyMeasured.pdf. Cited: Jan. 7, 2019.

Suber, P. Open access overview: focusing on open access to peer-reviewed research articles and their preprints. [S.I.S.n.], dez. 2015. Available from: http://legacy.earlham.edu/ peters/ fos/overview.htm. Cited: June 7, 2019.

Symplectic. A brief timeline of Open Access. [S.I.]: Sympletic, 2014. Infográfico. Available from: https://symplectic.co.uk/ open-access-timeline/. Cited: June 7, 2019. 
Text \& Academic Authors. Directory of Open Access Books $(D O A B)$ open to all publishers who publish academic, peer reviewed books in Open Access. Fountain City, USA, 8 Jul. 2018. Twitter: @TextandAcademic. Available from: https://twitter. com/TextandAcademic/status/1016004084858486791?s=20. Cited: May 25, 2019.

Torres-Salinas, D.; Robinson-Garcia, N.; Gorraiz, J. Filling the citation gap: measuring the multidimensional impacto of the academic book at institutional level with PlumX. Scientometrics, v.113, n.3, p.1371-1384, 2017. Available from: https://arxiv.org/ftp/arxiv/papers/1710/1710.00368.pdf. Cited: Jan. 11, 2019.

Twitter. Painel de atividades do tweet. [S.I.]:Twitter, 2018. Disponível em: https://business.twitter.com/pt/help/campaignmeasurement-and-analytics/tweet-activity-dashboard.html. Acesso em: 10 jan. 2019. 